\title{
Adaptive Fuzzy Routing in Opportunistic Network (AFRON)
}

\author{
Payam Nabhani \\ Department of Mathematics, Science and Research \\ Branch, Islamic Azad University, Khouzestan, Iran. \\ PO Box 163, Tee Airport Boulevard,Ahwaz, Iran
}

\author{
Amir Masoud Bidgoli \\ Department of Mathematics, Science and Research \\ Branch, Islamic Azad University, Khouzestan, Iran. \\ PO Box 163, Tee Airport Boulevard,Ahwaz, Iran
}

\begin{abstract}
Opportunistic network is a kind of Delay Tolerant Networks (DTN) where the nodes in this network come into contact with each other opportunistically and communicate wirelessly and, an end-to-end path between source and destination may have never existed, and disconnection and reconnection is common in the network. In such a network, because of the nature of opportunistic network, perhaps there is no a complete path from source to destination for most of the time and even if there is a path; the path can be very unstable and may change or break quickly. Therefore, routing is one of the main challenges in this environment and, in order to make communication possible in an opportunistic network, the intermediate nodes have to play important role in the opportunistic routing protocols. In this paper we proposed an Adaptive Fuzzy Routing in opportunistic network (AFRON). This protocol is using the simple parameters as input parameters to find the path to the destination node. Using Message Transmission Count, Message Size and Time To Live parameters as input fuzzy to increase delivery ratio and decrease the buffer consumption in the all nodes of network.
\end{abstract}

\section{General Terms}

Your general terms must be any term which can be used for general classification of the submitted material such as Pattern Recognition, Security, Algorithms et. al.

\section{Keywords}

Opportunistic Routing, Fuzzy Routing, Opportunistic Network, Message Routing.

\section{INTRODUCTION}

Opportunistic network is a type of Delay Tolerant Networks (DTN)[1] where network communication opportunities appear opportunistic, an end-to-end path between source and destination may have never existed, and disconnection and reconnection is common in the network. In the other words an opportunistic network as a subset of Delay-Tolerant Network where communication opportunities (contacts) are intermittent, so an end-to-end path between the source and the destination may never exist. The link performance in an opportunistic network is typically highly variable. Therefore, in the absence of reliable end to end connection between the source and destination node, TCP/IP protocol will not work. Opportunistic networking tries to simplify this aspect by providing several kinds of opportunistic routings. In opportunistic networks, communication devices can be carried by people, vehicles or animals, etc. Some devices can form a small mobile ad hoc network when the nodes move close to each other. But a node may frequently be isolated from other nodes. Therefore, a node is just intermittently connected to other nodes, and this partitioning is dynamically changing with time. Thus, an end-to-end connection between the source and the destination can be absent at the time the source wants to transmit, and even later.

Traditional routing protocols are not suitable for this scenario, because in those routing protocols, end-to-end connection between the source and the destination node is basic assumption. Devices in opportunistic network are enabled to interconnect by operating message in a store-carryforward style and, each node can act as host, intermediate node, thus, it can store, carry and forward the message between for other nodes. The big challenge in opportunistic networks is how to route messages from their source to their destination, with the absence of end-to-end path. When there is no path existing between the source and the destination, nodes need to communicate with each other via opportunistic contacts through store-carry-forward operation. This requires different routing approaches and schemes to cope with, on one side, and to exploit, on the other side, the characteristics of opportunistic networks. There are some important factors in routing message such as the role of context information as well as social aspects, where the classification of routing approaches is based on the amount of context information used as given in [2]. Based on the context information exploited, routing in opportunistic network is classified into three classes: context-oblivious, Partially Context-Aware and Fully Context-Aware Routing protocols. In this paper we proposed an Adaptive Fuzzy Routing which is used to use the all mentioned classes in opportunistic way to deliver the message according to the situations. The rest of this paper is organized as follow: section 2 addresses the opportunistic routing protocols. Section 3 describes AFRON protocol. Section 4 compares the AFRON with the other protocols and finally section 5 draws the conclusions.

\section{Related Work}

The routing in opportunistic network as mentioned before does not need to end-to-end path between the source and destination node and this fact is simplified the touting protocols in opportunistic networks; however, challenges remain that are distinct from those of conventional network routing methods. According to the classifications of routing protocols (i.e. context-oblivious, Partially Context-Aware and Fully Context-Aware)[2] there are some routing protocols have been proposed. In the context-oblivious, routing protocols are based on the flooding. To increase network capacity, the maximum number of repeated messages and the total number of copies of a message are limited. When nothing else is allowed to duplicate, the node should deliver the message directly to the final node. These protocols reduce the delay in getting the message, but many resources are consumed. These protocols are congestion and competition from other problems. Epidemic [3], Spray and wait [4], Fuzzy-Spray [15], MV [5] and Network Coding [6] are examples of flooding based protocols. The second class 
protocols attempt to gain a bit of background information, to improve their process. Defining algorithms to calculate the probability of delivering message to the destination node by using the background information is the main purpose of these protocols. PRoPHET[7], MaxProp[8], MoVe[9], MobySpace[10] and Bubble Rap[11] are some examples of Partially Context-Aware protocols and in the last class of routing protocols in opportunistic network based on background information is Fully Context-Aware. All protocols in this class are using the background information in order to deliver message to the destination node. These protocols provide the ability to adapt to different environments. HiBOp[12] and Propicman/SpatioTempo[2] are some example routing protocols this category.

\section{AFRON Protocol}

The Routing protocols in opportunistic networks, deal with knowledge and phenomena in many fields, which cannot be clearly just by true or false. Using fuzzy knowledge to process can improve the describing ability for fuzzy knowledge, and can grasp the essential meaning of fuzzy knowledge. For using the nature of opportunistic networks parameters such as the Message Transmitted Count of nodes, Message Size and Time To Live to calculate the importance of message. Due to ambiguity and uncertainty of opportunistic network environment a fuzzy based protocol is proposed to find the destination node in shortest time with less buffer usage. In this regards the following input and output sets and have been defined for AFRON protocol.

\subsection{Inputs Variables}

\subsubsection{Message Transmitted Count (MTC):}

In Fuzzy-Spray protocol [15], Forward Transmission Count or FTC was proposed in order to prioritize messages in buffer of nodes. In AFRON we used the same concept to calculate the copies of message in the network. This parameter is increased when the nodes exchanges their messages so it is approximately show the number of message transmission in the network. The value of MTC is as same as FTC but the membership function had been defined again and it is depicted in Fig.1.

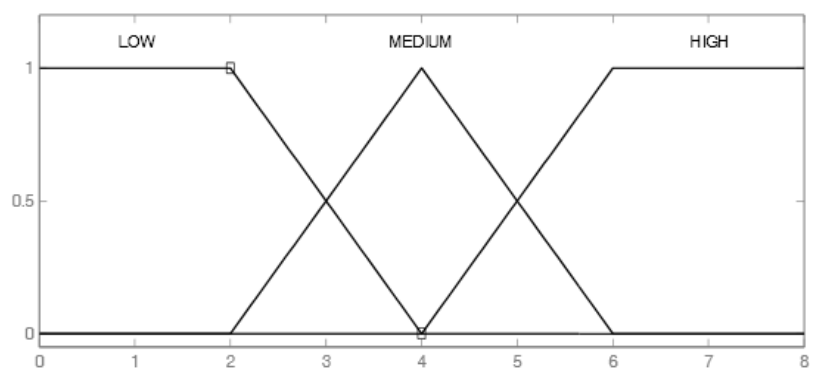

Fig.1: MTC Membership function

\subsubsection{Message Size(MS):}

Since that most nodes of the mobile network nodes or devices have limited buffer size and the size of message is very important so we considered it as input parameters as same as Fuzzy Spray Protocol but there is some deference in the member ship function which is defined as Fig. 2 .

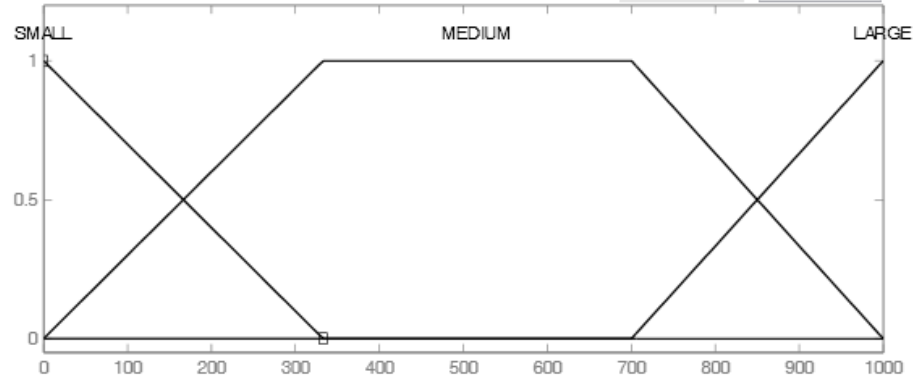

Fig.2:MS Membership Function

\subsubsection{Time To Live (TTL):}

In fact the TTL time is very important in routing protocol. When messages are not delivered in their TTL, the drop ration of message will be increased and the total performance of proto9cl will be decreased. TTL is considered in our protocol in order to increasing the ratio of message delivery which is not considered in Fuzzy-Spray protocol. The membership functions for TTL is depicted in Fig. 3

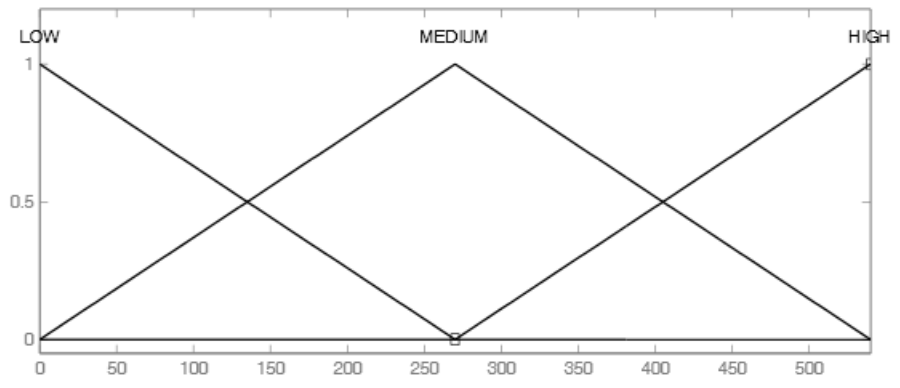

Fig. 3: TTL Membership Function

\subsection{Output Variable (Buffer Sections):}

According to the input, AFRON protocol will be divide the buffer of nodes into 19 sections and according to the input the message will be select the appropriate section. This partitioning finally will be used to prioritize the message in order to exchange it in next contact. The priority of message will be calculated as bellow:

Priority_of_Message=1-BS_of_Message.

Error! Reference source not found. shoes the membership function of BS

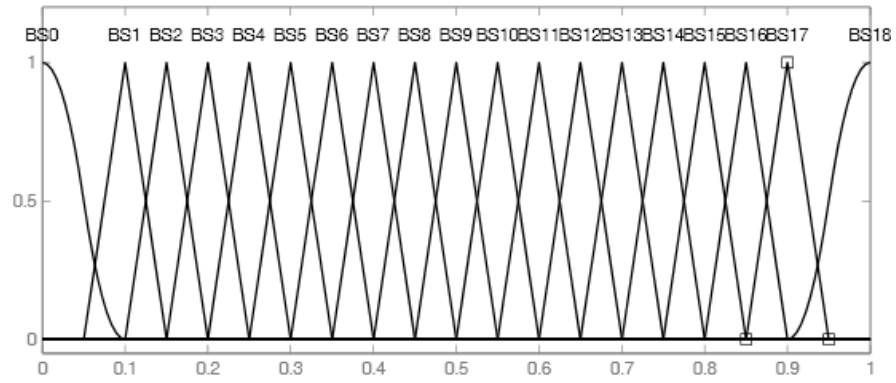

Table 1Fig. 3: TTL Membership Function 


\subsection{Fuzzy Rules:}

In AFRON protocol Fuzzy Rules are defined to prioritize the messages in order to deliver the message in shortest time and before the end of message's TTL. Error! Reference source not found. is shows all fuzzy rules in AFRON protocol.

Table 2. FUZZY RULES IN AFRONP ROTOCOL

\begin{tabular}{|c|c|c|c|}
\hline MTC & MS & TTL & BS \\
\hline low & small & low & BSO \\
\hline low & small & medium & BSO \\
\hline low & small & high & BSO \\
\hline low & medium & low & BS1 \\
\hline low & medium & medium & BS1 \\
\hline low & medium & high & BS2 \\
\hline low & large & low & BS3 \\
\hline low & large & medium & BS3 \\
\hline low & large & high & BS4 \\
\hline medium & small & low & BS4 \\
\hline medium & small & medium & BS4 \\
\hline medium & small & high & BS5 \\
\hline medium & medium & low & BS6 \\
\hline medium & medium & medium & BS7 \\
\hline medium & medium & high & BS8 \\
\hline medium & large & low & BS9 \\
\hline medium & large & medium & BS9 \\
\hline medium & large & high & BS10 \\
\hline high & small & low & BS11 \\
\hline high & small & medium & BS11 \\
\hline high & small & high & BS12 \\
\hline high & medium & low & BS13 \\
\hline high & medium & medium & BS14 \\
\hline high & medium & high & BS15 \\
\hline high & large & low & BS16 \\
\hline high & large & medium & BS17 \\
\hline
\end{tabular}

\begin{tabular}{|c|c|c|c|}
\hline high & large & high & BS18 \\
\hline
\end{tabular}

\subsection{AFRON Algorithm:}

The following Pseudo-code is shows how the AFRON is working. It is very similar to the Fuzzy-Spray and Spray and

Wait Protocol.

AdaptiveFuzzy()

\{

/*Node $\mathrm{i}$ is connected to Node $\mathrm{j}$ /

while(NodesAreConnected(i,j)\{

${ }^{*}$ send all message from node i buffer to node $j^{*} /$

SendMessage(i,j);

${ }^{*}$ send all message from node i buffer to node $j^{*} /$

SendMessage(j,i);

\}

\section{\}//end of AdaptiveFuzzy}

SendMessage(i,j) \{

${ }^{*}$ deliver all messages in node $i$ that their destinations is node $j^{*}$ /

DeliveredtoFinalNode (i,j);

${ }^{*}$ each node send Ack to the other node in case of delivering $* /$

ExchangeAck $(i, j)$

${ }^{*}$ set the priority of message in buffer of node $i^{*} /$

SetMessagesPriority (i);

${ }^{*}$ node I and $j$ exchange the message in their buffer*/

ExchangeMessage (i, j);

${ }^{*}$ remove the delivered messages from the buffers of both nodes $i$ and $j^{*} /$

RemoveDeliveredMessage $(i, j)$ 


\section{\}//end of SendMessage \\ SetMessagesPriority (i) \\ \{ \\ for each message in $_{i}$ Buffer ${ }_{i}\{$ \\ if IsFoundIn(message ${ }_{i}$, Ack $\left._{i}\right)$ then \\ remove $\left(\operatorname{message}_{i}\right)$ \\ else}

message $_{i}$. priority=1-

Fuzzification(message ${ }_{i}$ )

\}

SortByPriority(Buffer B $_{i}$

\}//end of SetMessagesPriority

\section{Simulation Results}

The ONE [16][17] is used for simulation. There scenarios are defined in order to compare the message delivery ratio and buffer consumption of AFRON protocols with the other same protocols such as Fuzzy-Spray, Spray and Wait and Epidemic.

The simulation time is set to 12 Hours and the communication interface is Bluetooth with $10 \mathrm{~m}$ rang. The transmission speed is $25 \mathrm{~KB} / \mathrm{s}$.

\subsection{Levy Walk[18] Scenario:}

The scenarios are based on the internal movement of the nodes. Nodes are divided into two groups. The first group moving model is StationaryMovement model and the second movement model is Community model. The simulation area is $400 * 200$ square meters and the messages are generated randomly by using Exponential distribution function. The interval time between two messages is $10-100$ seconds. The buffer size is $100 \mathrm{MB}$ and the message size is $100-200 \mathrm{~KB}$.

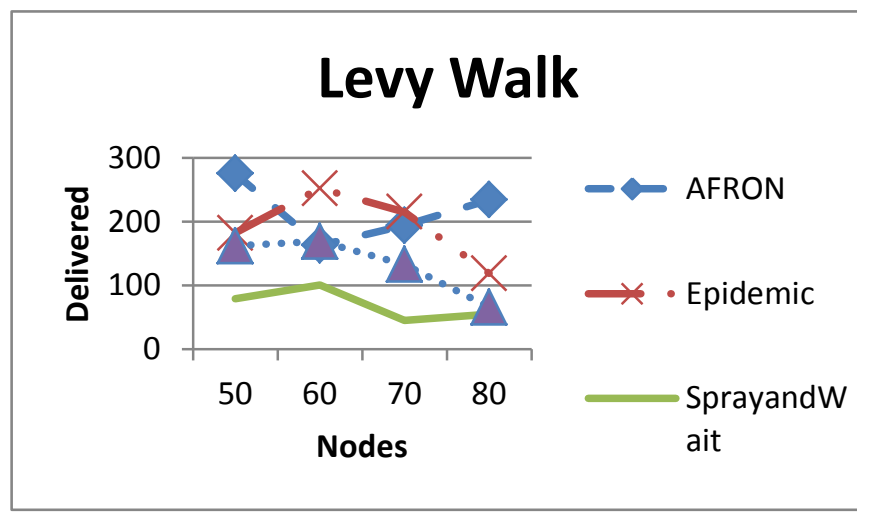

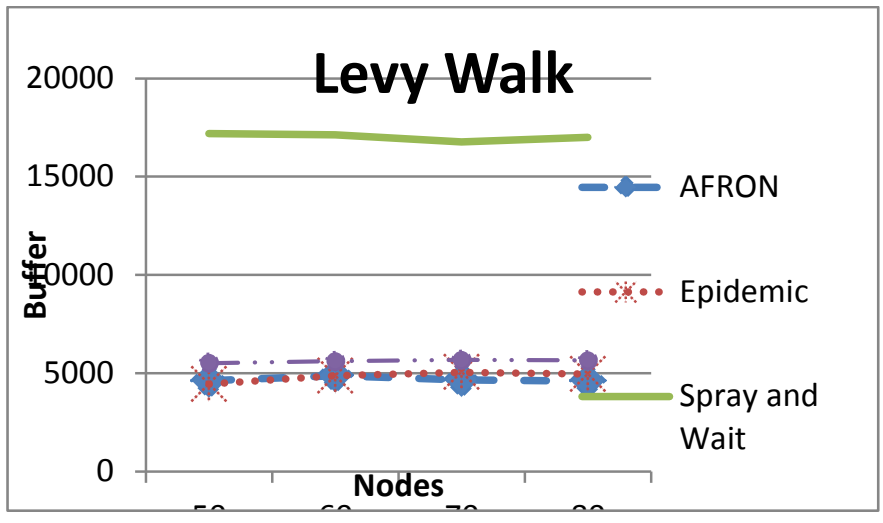

Fig. 5: Buffer Consumption for Levy Walk Scenario

\subsection{Random Walk Scenario:}

In this scenario all settings are the same as the first scenario except the internal movement which is here set to Random Walk movement model.

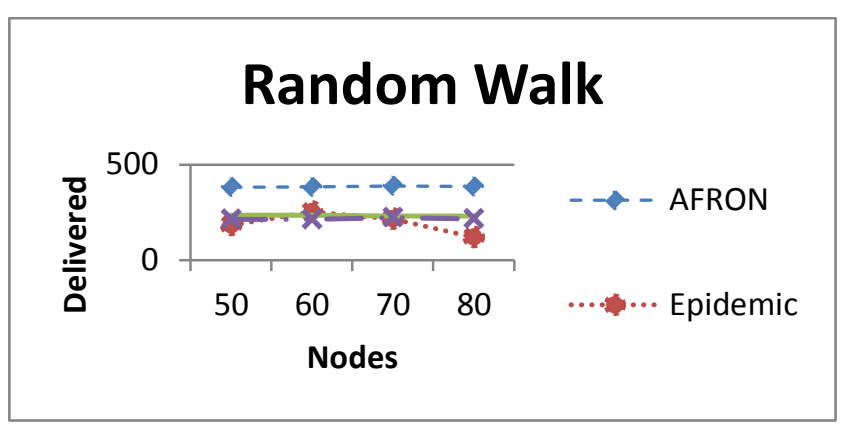

Fig. 6: Message Delivery for Random Walk Scenario

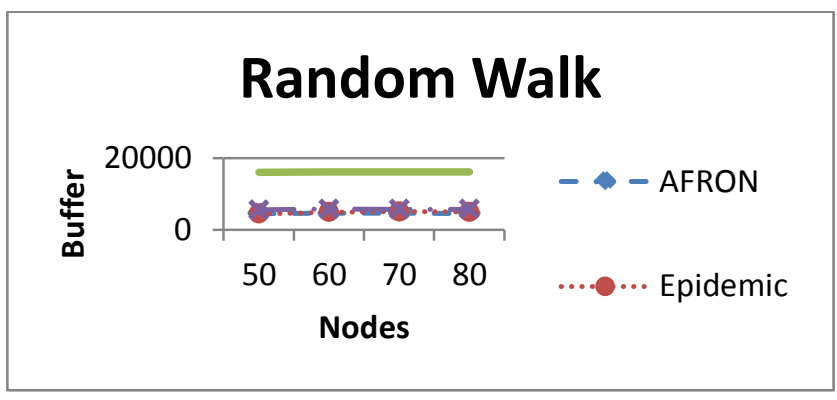

Fig. 7: Buffer Consumption for Random Walk Scenario

\subsection{Random Waypoint Scenario:}

All setting are the same as the first but the internal movement is Random Waypoint model.

Fig. 4:Message Delivery for Levy Walk Scenario 


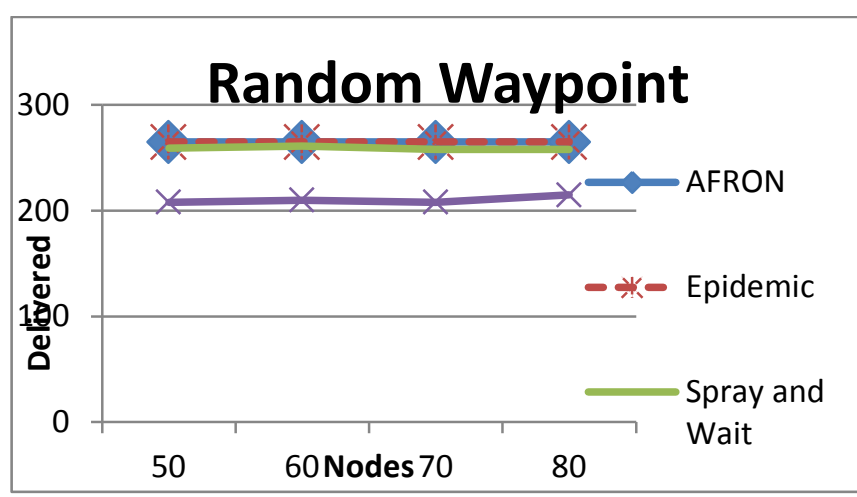

Fig. 8: Message Delivery for Random Waypoint Scenario

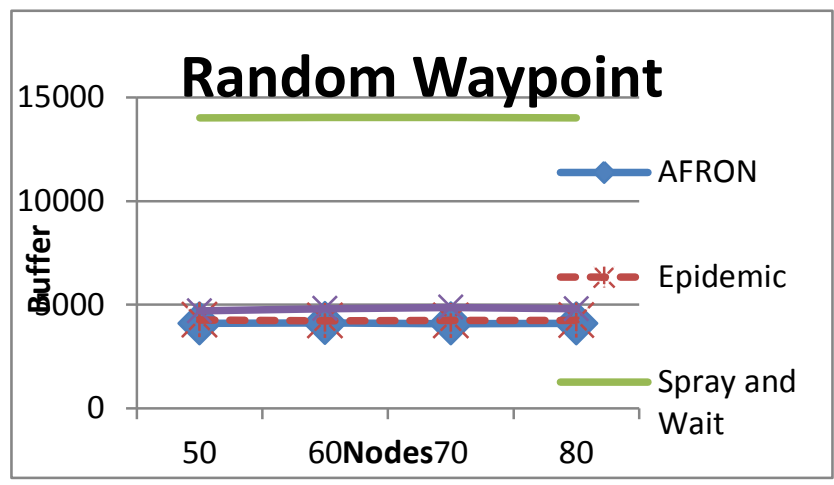

Fig. 9: Buffer Consumption for Random Waypoint Scenario

As shown above the AFRON is working better than the other protocols especially in the random movement of nodes

\section{Conclusion}

This paper has presented a new method to dynamically select the forwarding list according to the situation. The algorithm is supported by an anycast transmission technique. This mechanism to select the relaying node from the available node list is supported by a fuzzy logic system which takes into account the bandwidth, energy of the node, priority of the message and the density of the network. By means of fuzzy logic, the effectiveness of AFRON protocol is able to reduce the energy consumption per transmission and also could use less resource. As future work, we intend to implement the protocol in a network simulation where realistic propagation conditions and realistic battery performance are taken into account.

\section{REFERENCES}

[1] Delay Tolerant Networking Research Group. http://www.dtnrg.org.

[2] Conti, M., Crowcroft, J., Giordano, S., Hui, P., Nguyen, H.A., \& Passarella, A.(2008). Minema. Hugo Miranda, Luis Rodrigues,Benoit Garbinato (Ed.), "Routing issues in Opportunistic Networks". Springer.

[3] Vahdat, A., \& Becker, D.(2000, April). "Epidemic Routing for Partially Connected Ad hoc Networks" (Tech. Rep. CS-200006). Duke University.

[4] T. Spyropoulos, k. Psounis, C.S. Raghavendra, "Spray and wait: An efficient routing scheme for intermittently connected mobile networks", ACM SIGCOMM Workshop on Delay-Tolerant Networking 2005, pp. 252259, 2005.

[5] B. Burns, O. Brock, and B. N. Levine, "MV Routing and capacity building in disruption tolerant networks", Proceedings of the IEEE INFOCOM 2005, Miami, FL, March, 2005.

[6] J. Widmer and J.-Y. Le Boudec, "Network Coding for Efficient Communication in Extreme Networks", Proceedings of the ACM SIGCOMM 2005 Workshop on delay tolerant networks, Philadelphia, PA, USA, August 22-26, 2005.

[7] A. Lindgren, A. Doria, and O. Schelèn, "Probabilistic routing in intermittently connected networks", Mobile Computing and Communications Review, 7(3), July, 2003.

[8] J. Burgess, B. Gallagher, D. Jensen and B. N. Levine, "MaxProp: Routing for Vehicle-Based DisruptionTolerant Networks," Proceedings of 25th IEEE International Conference on Computer Communications, Barcelona, 23-29 April 2006, pp. 1-11. doi:10.1109/INFOCOM.2006. 228

[9] J. LeBrun, C.-N. Chuah, D. Ghosal, and M. Zhang, "Knowledgebased opportunistic forwarding in vehicular wireless ad hoc networks," In IEEE Vehicular Technology Conference(VTC), pp. 2289-2293, May 2005.

[10] J. Leguay, T. Friedman, V. Conan, "DTN Routing in a Mobility Pattern Space", presented at ACM SIGCOMM Workshop on Delay Tolerant Networking, 2005

[11] Hui, P. and Crowcroft, J. (2007) "Bubble rap: forwarding in small world dtns in every decreasing circles", Technical report, Technical Report UCAM-CL-TR684. Cambridge, UK: University of Cambridge.

[12] Boldrini, C., Conti, M., Jacopini, I., \& Passarella, A.(2007, June). "HiBOp: A History Based Routing Protocol for Opportunistic Networks". Paper presented in the Proceedings of the WoWMoM 2007, Helsinki.

[13] Hemal Shah and Yogeshwar. P. Kosta , "Routing Enhancement Specific to Mobile Environment Using DTN", International Journal of Computer Theory and Engineering, Vol. 3, No. 4, August 2011

[14] Chung-Ming Huang, Kun-chan Lan and Chang-Zhou Tsai ,"A Survey of Opportunistic Networks", 22nd International Conference on Advanced Information Networking and Applications - Workshops 978-0-76953096-3/08 \$25.00 @ 2008 IEEE

[15] A. Mathurapoj, C. Pornavalai, "Fuzzy-Spray: Efficient Routing in Delay Tolerant Ad-hoc Network Based on Fuzzy Decision Mechanism", Fuzzy Systems, 2009. FUZZ-IEEE Aug. 2009 pp: 104 - 109.

[16] http://www.netlab.tkk.fi/tutkimus/dtn/theone [on-line 2012]

[17] Ari Keränen, Jörg Ott, Teemu Kärkkäinen, "The ONE Simulator for DTN Protocol Evaluation", SIMUTools 2009, Rome, Italy.

[18] http://en.wikipedia.org/wiki/Levy_walk [on line 2012] 